\title{
Simulating a toy model of electrodynamics in $(1+1)$ dimensions
}

\author{
A. D. Boozer ${ }^{a}$ \\ Department of Physics, California Institute of Technology, Pasadena, California 91125
}

(Received 4 August 2008; accepted 10 November 2008)

\begin{abstract}
We show how to simulate a toy model of electrodynamics in $(1+1)$ dimensions and describe several numerical experiments. The toy model is much simpler than ordinary electrodynamics, but shares many of the same physical features. For example, there are analogs to the electric and magnetic fields, and these fields generate forces between charged particles and support freely propagating radiation. Unlike electrodynamics, however, the toy model is not Lorentz invariant, gives an attractive force between charges of the same sign, and yields a radiation reaction force that depends on the particle velocity. (0) 2009 American Association of Physics Teachers.
\end{abstract}

[DOI: 10.1119/1.3040670]

\section{INTRODUCTION}

The theory of radiation in classical electrodynamics is mathematically complicated and conceptually subtle. For example, it requires a lengthy calculation to find the retarded electric and magnetic fields of a charged particle in arbitrary motion, and problems such as radiation reaction touch on deep foundational issues. The combination of mathematical and conceptual obstacles can make the subject difficult for the beginning student to understand.

To help overcome these obstacles we show how a broad range of radiative phenomena can be illustrated by performing computer simulations of a toy model of electrodynamics in $(1+1)$ dimensions. ${ }^{1}$ The toy model is a useful pedagogical tool, because it is much simpler than ordinary electrodynamics but shares many of the same physical features. For example, there are analogs to the electric and magnetic fields, and these fields generate forces between charged particles and support freely propagating radiation. The toy model is particularly well suited to illustrating radiative phenomena, and can be used to introduce concepts such as retarded fields, radiation damping, and scattering. By performing simulations of the toy model, students can see how these concepts operate in a simplified setting. ${ }^{2}$ The paper is intended to supplement a discussion of radiation in an undergraduate or graduate level electrodynamics class, and should be accessible to advanced undergraduates.

The paper is organized as follows. In Sec. II we describe the toy model and show how it is related to ordinary electrodynamics. In Sec. III we discretize the model so it can be simulated on a computer. In Sec. IV we describe four numerical experiments that can be performed using the simulation. In Sec. V we discuss several advanced topics pertaining to spatially extended charged particles.

\section{TOY MODEL OF ELECTRODYNAMICS}

The toy model that we will consider describes a point particle in $(1+1)$ dimensions that obeys Newtonian dynamics and is coupled to a pair of fields $E(x, t)$ and $B(x, t){ }^{3}$ The equations of motion for these fields are ${ }^{4}$

$$
\begin{aligned}
& \partial_{t} E(x, t)=\partial_{x} B(x, t), \\
& \partial_{t} B(x, t)=\partial_{x} E(x, t)-2 \rho(x, t),
\end{aligned}
$$

where $\rho(x, t)$ is the charge density. We will assume that the charge density has the form

$$
\rho(x, t)=g \delta(x-z(t)),
$$

where $z(t)$ is the position of the particle at time $t$ and $g$ is its charge. The equation of motion for the particle is

$$
m \ddot{z}=F_{\mathrm{ext}}+F_{f},
$$

where $m$ is the mass of the particle, $F_{\text {ext }}$ is an externally imposed force, and

$$
F_{f}(t)=-2 g E(z(t), t)
$$

is the force that the $E$-field exerts on the particle. Equations (1)-(5) give a complete description of the model. It is possible to derive Eqs. (1)-(5) from more fundamental principles, ${ }^{3}$ but here we will take them as given. Each equation has a analog in ordinary electrodynamics; for example, Eqs. (1) and (2) are analogous to Maxwell's equations, and Eq. (5) is analogous to the Lorentz force law. ${ }^{5}$ Also, the $E$ and $B$ fields are analogous to the electric and magnetic fields.

We can think of the $E$ and $B$ fields as each consisting of two components: one that is generated by the particle, and one that describes any freely propagating radiation that is present. It is useful to decompose the $E$ and $B$ fields so as to separate these components:

$$
\begin{aligned}
& E(x, t)=E_{r}(x, t)+E_{\text {in }}(x, t), \\
& B(x, t)=B_{r}(x, t)+B_{\text {in }}(x, t),
\end{aligned}
$$

where $E_{r}(x, t)$ and $B_{r}(x, t)$ describe the retarded fields generated by the particle, and $E_{\text {in }}(x, t)$ and $B_{\text {in }}(x, t)$ describe incoming radiation. ${ }^{6}$ For events that do not lie on the worldline of the particle (that is, events $(x, t)$ such that $x \neq z(t)$ ), the retarded fields are given by ${ }^{7,8}$

$$
\begin{aligned}
& E_{r}(x, t)=\frac{g \epsilon\left(x-z\left(t_{r}\right)\right)}{1-v\left(t_{r}\right) \epsilon\left(x-z\left(t_{r}\right)\right)}, \\
& B_{r}(x, t)=-\frac{g v\left(t_{r}\right) \epsilon\left(x-z\left(t_{r}\right)\right)}{1-v\left(t_{r}\right) \epsilon\left(x-z\left(t_{r}\right)\right)} .
\end{aligned}
$$

Here $z\left(t_{r}\right)$ and $v\left(t_{r}\right)$ are the position and velocity of the particle at the retarded time $t_{r}(x, t)$, which is defined implicitly by $t_{r}=t-\left|x-z\left(t_{r}\right)\right|$. For events that lie on the worldline of the particle (that is, events $(x, t)$ such that $x=z(t))$, the retarded fields are given by 


$$
\begin{aligned}
& E_{r}(z(t), t)=g v(t)\left(1-v^{2}(t)\right)^{-1}, \\
& B_{r}(z(t), t)=-g v^{2}(t)\left(1-v^{2}(t)\right)^{-1} .
\end{aligned}
$$

The retarded fields satisfy the equations of motion

$$
\begin{aligned}
& \partial_{t} E_{r}(x, t)=\partial_{x} B_{r}(x, t), \\
& \partial_{t} B_{r}(x, t)=\partial_{x} E_{r}(x, t)-2 \rho(x, t),
\end{aligned}
$$

so from Eqs. (1), (2), (6), and (7), it follows that the in-fields satisfy the equations of motion

$$
\begin{aligned}
& \partial_{t} E_{\text {in }}(x, t)=\partial_{x} B_{\text {in }}(x, t), \\
& \partial_{t} B_{\text {in }}(x, t)=\partial_{x} E_{\text {in }}(x, t) .
\end{aligned}
$$

Equations (14) and (15) imply that the in-fields satisfy the wave equation, and therefore describe freely propagating radiation:

$$
\left(\partial_{t}^{2}-\partial_{x}^{2}\right) E_{\text {in }}(x, t)=\left(\partial_{t}^{2}-\partial_{x}^{2}\right) B_{\text {in }}(x, t)=0 .
$$

As an example, consider a stationary particle at position $z_{0}$. From Eqs. (8) and (9) we find that the retarded fields are $E_{r}(x, t)=g \epsilon\left(x-z_{0}\right)$ and $B_{r}(x, t)=0$. These are just the static fields of the stationary charge, and are analogous to the Coulomb fields $\vec{E}(\vec{r}, t)=e \hat{r} / r^{2}, \vec{B}(\vec{r}, t)=0$ of a stationary point charge in ordinary electrodynamics.

If we use the decomposition of the $E$-field given in Eq. (6), we can express the force law in Eq. (5) as $F_{f}=F_{\text {in }}+F_{r}$, where

$$
F_{\text {in }}(t)=-2 g E_{\text {in }}(z(t), t)
$$

is the force exerted on the particle by the incoming radiation, and

$$
F_{r}(t)=-2 g E_{r}(z(t), t)
$$

is the force exerted on the particle by its own retarded field. If we substitute for $E_{r}(z(t), t)$ using Eq. (10) and define a damping constant $\gamma=2 \mathrm{~g}^{2} / \mathrm{m}$, we find that

$$
F_{r}=-m \gamma \dot{z}\left(1-\dot{z}^{2}\right)^{-1} \text {. }
$$

The force $F_{r}$ is analogous to the radiation reaction force of ordinary electrodynamics. In the toy model a moving particle radiates, and $F_{r}$ describes the force that the emitted radiation exerts back on the particle. ${ }^{9}$ By using Eq. (19) we can express the equation of motion (4) in the form

$$
m \ddot{z}+m \gamma \dot{z}\left(1-\dot{z}^{2}\right)^{-1}=F_{\text {ext }}+F_{\text {in }} .
$$

Note that by replacing Eqs. (1), (2), and (4) with Eqs. (14), (15), and (20), we have eliminated the retarded fields from our description of the system.

We will often work in the limit in which the speed of the particle is much less than the speed at which waves are propagated by the field $(|\dot{z}| \ll 1)$. In this limit the equation of motion reduces to

$$
m \ddot{z}+m \gamma \dot{z}=F_{\text {ext }}+F_{\text {in }} .
$$

So far we have considered a single particle, but it is straightforward to generalize the model to multiple particles. We find that charges of the same sign attract. To see why, consider two particles with charges $g_{1}$ and $g_{2}$ and positions $z_{1}$ and $z_{2}$. The static $E$-field generated by particle one is $E_{1}(x, t)=g_{1} \epsilon\left(x-z_{1}\right)$, and the force exerted on particle two is

$$
F_{2}=-2 g_{2} E_{1}\left(z_{2}, t\right)=-2 g_{1} g_{2} \epsilon\left(z_{2}-z_{1}\right) \text {. }
$$

There are several important differences between the toy model and ordinary electrodynamics. ${ }^{10}$ One difference is that in ordinary electrodynamics charges of the same sign feel a repulsive force, but in the toy model they feel an attractive force. Another difference is that ordinary electrodynamics is Lorentz invariant, whereas the toy model is neither Galilean nor Lorentz invariant. ${ }^{11}$ Also, in ordinary electrodynamics the radiation reaction force is proportional to the time derivative of the acceleration, whereas in the toy model it depends on the velocity. These differences are intrinsic to the model we are considering, which was constructed to illustrate radiative phenomena. In Appendix A we describe electrodynamics in $(1+1)$ dimensions; this theory is Lorentz invariant and gives a repulsive force between like charges, but does not exhibit radiation.

Despite these differences, the toy model shares much of the conceptual structure of ordinary electrodynamics. As we have shown, there are direct analogs to the electric and magnetic fields, Maxwell's equations, and the Lorentz force law. We also have shown that the coupling of the particle to the $E$ and $B$ fields allows it to emit radiation, and we have derived analogs to the retarded fields and to the radiation reaction force. As we shall see, these features allow us to use the toy model to illustrate a broad range of radiative phenomena.

\section{NUMERICAL SIMULATION}

To simulate the toy model we will make three modifications to the theory presented in Sec. II: we will express the theory in terms of a scalar potential, generalize the theory to the case of a spatially extended particle, and discretize the theory so that it can be described using a finite number of degrees of freedom.

In ordinary electrodynamics the two homogeneous Maxwell equations imply that we can express the electric and magnetic fields as derivatives of a vector potential $A^{\mu}(\vec{r}, t)$. Similarly, in the toy model Eq. (1) implies that we can express the $E$ and $B$ fields as derivatives of a scalar potential $\phi(x, t)$ :

$$
E(x, t)=\partial_{x} \phi(x, t), \quad B(x, t)=\partial_{t} \phi(x, t) .
$$

We can use Eq. (2) to express the equations of motion for the field variables $\phi(x, t)$ and $B(x, t)$ as

$$
\begin{aligned}
& \partial_{t} \phi(x, t)=B(x, t), \\
& \partial_{t} B(x, t)=\partial_{x}^{2} \phi(x, t)-2 \rho(x, t) .
\end{aligned}
$$

We can express the force law as

$$
F_{f}(t)=-\left.2 g \partial_{x} \phi(x, t)\right|_{x=z(t)} .
$$

Before we can discretize the toy model, we must first generalize it to the case of a spatially extended particle. We replace the charge density given by Eq. (3) with

$$
\rho(x, t)=g f(x-z(t)),
$$

where the function $f(x)$ describes the charge distribution of the particle, which is taken to be rigid. In what follows we will assume that $f(x)$ is a Gaussian: 


$$
f(x)=\left(2 \pi \sigma^{2}\right)^{-1 / 2} e^{-x^{2} / 2 \sigma^{2}},
$$

where $\sigma$ characterizes the particle size. We also need to modify the force law given by Eq. (5). For an extended particle the force law is

$$
F_{f}(t)=-2 \int \rho(x, t) E(x, t) d x .
$$

For $f(x)=\delta(x)$ Eq. (29) reduces to the force law for a point particle given by Eq. (5). We substitute for $E(x, t)$ and $\rho(x, t)$ using Eqs. (23) and (27) and then integrate by parts to find that

$$
F_{f}(t)=2 g \int \phi(x, t) f^{\prime}(x-z(t)) d x,
$$

where $f^{\prime}(x) \equiv d f(x) / d x=-\left(x / \sigma^{2}\right) f(x)$. From Eqs. (4) and (30) it follows that the equation of motion for an extended particle is

$$
m \ddot{z}=F_{\text {ext }}+2 g \int \phi(x, t) f^{\prime}(x-z(t)) d x .
$$

To discretize the toy model we introduce a spatial lattice and transfer the continuum theory to the lattice by making a series of replacements. We label the lattice sites by an integer $n$, denote the distance between adjacent sites by $a$, and define a frequency $\omega_{F} \equiv 1 / a$. We replace the spatial coordinate $x$ by $a n$, and replace integrals over $x$ with sums over $n$. We define a lattice scalar potential $\phi_{n}(t)$ by $\phi(x, t) \rightarrow a \phi_{n}(t)$, lattice fields $E_{n}(t)$ and $B_{n}(t)$ by $E(x, t) \rightarrow E_{n}(t)$ and $B(x, t) \rightarrow B_{n}(t)$, and a lattice charge density $\rho_{n}(t)$ by

$$
\rho(x, t) \rightarrow \rho_{n}(t)=g f(a n-z(t)) .
$$

The spatial derivatives of $\phi(x, t)$ are replaced as follows:

$$
\begin{aligned}
& \partial_{x} \phi(x, t) \rightarrow\left(\phi_{n+1}(t)-\phi_{n-1}(t)\right) / 2, \\
& \partial_{x}^{2} \phi(x, t) \rightarrow\left(\phi_{n+1}(t)-2 \phi_{n}(t)+\phi_{n-1}(t)\right) / a .
\end{aligned}
$$

After making these replacements, we find that $E_{n}(t)$ and $B_{n}(t)$ are given by

$$
\begin{aligned}
& E_{n}(t)=(1 / 2)\left(\phi_{n+1}(t)-\phi_{n-1}(t)\right), \\
& B_{n}(t)=a \dot{\phi}_{n}(t) .
\end{aligned}
$$

The equations of motion (24) and (25) for $\phi(x, t)$ and $B(x, t)$ become

$$
\begin{aligned}
& \dot{\phi}_{n}=\omega_{F} B_{n}, \\
& \dot{B}_{n}=\omega_{F}\left(\phi_{n+1}-2 \phi_{n}+\phi_{n-1}\right)-2 \rho_{n},
\end{aligned}
$$

and the particle equation of motion Eq. (31) becomes

$$
m \ddot{z}=F_{\mathrm{ext}}+2 g a^{2} \sum_{n} \phi_{n} f^{\prime}(a n-z) .
$$

Equations (32) and (37)-(39) define the discretized version of the toy model and approximate the continuum theory of a point particle presented in Sec. II. The approximation is justified if the particle size $\sigma$ is large compared to the lattice spacing $a$, but small enough that the particle can still be treated as pointlike. In Sec. V we discuss the conditions that $\sigma$ must satisfy for the point particle approximation to apply. For now we note that for the numerical experiments presented in Sec. IV we choose $\sigma$ such that these conditions are met.

\section{A. Example solutions}

We now consider some example solutions to the discretized version of the toy model. From Eqs. (37) and (38) we find that

$$
\ddot{\phi}_{n}=\omega_{F}^{2}\left(\phi_{n+1}-2 \phi_{n}+\phi_{n-1}\right)-2 \omega_{F} \rho_{n} .
$$

Let us first consider the solutions to Eq. (40) when no charge is present $\left(\rho_{n}=0\right)$. We can write the solutions in the form

$$
\phi_{n}(t)=\left(\omega_{F} / \omega\right) \operatorname{Re}\left[A e^{i(k a n-\omega t)}\right],
$$

where $k$ is an arbitrary real number, $A$ is an arbitrary complex number, and

$$
\omega=2 \omega_{F}|\sin (k a / 2)| \text {. }
$$

These solutions describe monochromatic waves with wave vector $k$ and frequency $\omega$.

From now on we will only consider waves for which the wavelength is long compared to the lattice spacing ( $|k a|$ $\ll 1)$. For such waves Eq. (42) reduces to $\omega \simeq|k|$, which is the correct dispersion law for the continuum theory. In the long-wavelength limit the fields corresponding to $\phi_{n}(t)$ are

$$
\begin{aligned}
& B_{n}(t)=\operatorname{Im}\left[A e^{i(k a n-\omega t)}\right], \\
& E_{n}(t)=-\epsilon(k) B_{n}(t) .
\end{aligned}
$$

We can also solve Eq. (40) for the static potential generated by a time-independent charge distribution $\rho_{n}$ :

$$
\phi_{n}=a \sum_{r}|n-r| \rho_{r} .
$$

Equation (45) is useful for obtaining initial conditions. To integrate the equations of motion for the coupled particlefield system we need initial conditions for $\phi_{n}$ and $B_{n}$. We will often take these to be the static potential and $B$-field of a stationary particle at the origin:

$$
\phi_{n}(0)=g a \sum_{r}|n-r| f(a r), \quad B_{n}(0)=0 .
$$

The static field $E_{n}(0)$ can be obtained from $\phi_{n}(0)$ by using Eq. (35). In Fig. 1 we plot the static fields for a particle of size $\sigma=5$, where we have chosen a system of units such that $g=a=1$.

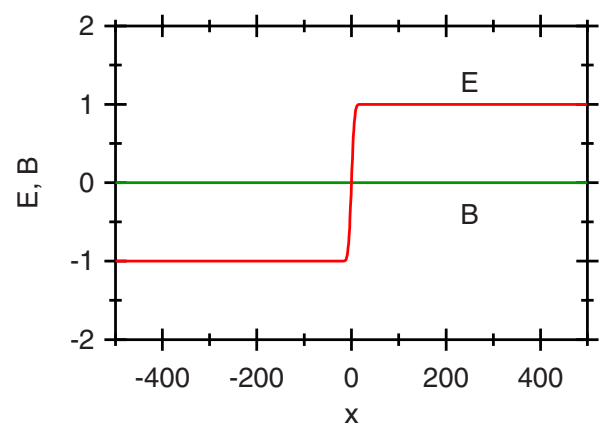

Fig. 1. Static $E$ and $B$ fields for a stationary particle. 


\section{B. Boundary conditions}

To simulate the discretized theory on a computer we will restrict the theory to a finite lattice and impose boundary conditions on the terminal lattice sites so that outgoing radiation is absorbed. We will assume that the lattice index $n$ ranges from $-R$ to $R$, so the total number of lattice sites is $N=2 R+1$. If the lattice extended beyond site $R$, the equation of motion for $B_{R}$ would be given by Eq. (38):

$$
\dot{B}_{R}=\omega_{F}\left(\phi_{R+1}-2 \phi_{R}+\phi_{R-1}\right)
$$

where we have assumed that the particle is far away from the edge of the lattice, so $\rho_{R}=0$. If we replace Eq. (47) with an equation of motion that gives the same time evolution for $B_{R}$, but which does not contain the variable $\phi_{R+1}$, then waves incident on site $R$ will be absorbed. We can obtain such an equation as follows.

Let us assume that the radiation incident on site $R$ takes the form of a right-moving monochromatic wave. If no charge is present, then Eqs. (35) and (44) imply that

$$
E_{R}=(1 / 2)\left(\phi_{R+1}-\phi_{R-1}\right)=-B_{R} .
$$

If we now assume that there is a total quantity of charge $g$, then this result must be modified to take into account the static fields of the charge. The static fields at site $R$ are given by $E_{R}=g$ and $B_{R}=0$, so we replace Eq. (48) with

$$
(1 / 2)\left(\phi_{R+1}-\phi_{R-1}\right)-g=-B_{R} \text {. }
$$

From Eq. (49) it follows that $\phi_{R+1}=\phi_{R-1}+2\left(g-B_{R}\right)$. We substitute this relation into Eq. (47) and find that

$$
\dot{B}_{R}=2 \omega_{F}\left(\phi_{R-1}-\phi_{R}-B_{R}+g\right) \text {. }
$$

We can derive an analogous equation of motion for $B_{-R}$ :

$$
\dot{B}_{-R}=2 \omega_{F}\left(\phi_{-R+1}-\phi_{-R}-B_{-R}+g\right) .
$$

To describe scattering phenomena it is useful to have boundary conditions that absorb outgoing radiation and produce incoming radiation. To produce a right-moving wave with amplitude $A$ and frequency $\omega$ that originates from the leftmost lattice site $-R$, we replace Eq. (51) with

$$
\dot{B}_{-R}=2 \omega_{F}\left(\phi_{-R+1}-\phi_{-R}-B_{-R}+g\right)+4 \omega_{F} \operatorname{Im}\left[A e^{-i \omega(a R+t)}\right] .
$$

It is straightforward to verify that Eq. (52) produces a wave with the desired properties by substituting for $\phi_{n}$ and $B_{n}$ using Eqs. (41) and (43). Note that if we take the initial conditions for $\phi_{n}$ and $B_{n}$ to be the static fields of a stationary charge and use Eqs. (50) and (52) to define the boundary conditions, we can simulate a system in which the in-fields describe incoming radiation that approaches the particle from the left.

\section{NUMERICAL EXPERIMENTS}

We now will use the discretized model to perform a series of numerical experiments that simulate the time evolution of the coupled particle-field system. For each experiment we first choose initial conditions for the variables $z, v, \phi_{n}$, and $B_{n}$. We then evolve the system by numerically integrating the equations of motion Eqs. (37)-(39), (50), and (51). ${ }^{12} \mathrm{We}$ choose a system of units such that $g=a=1$, and set $\sigma=5$. Unless otherwise noted, we assume that the particle moves slowly compared to the speed of wave propagation $(|\dot{z}| \ll 1)$.

\section{A. Harmonically bound particle}

We first consider a harmonically bound particle. ${ }^{13}$ The external force is $F_{\text {ext }}=-m \omega_{0}^{2} z$, where $\omega_{0}$ is the harmonic frequency. We assume that for $t<0$ the particle is at rest at the origin. Also, we assume that there is no incoming radiation $\left(E_{\text {in }}=B_{\text {in }}=0\right)$, so for $t<0$ the $E$ and $B$ fields are just the static fields of the stationary particle. At $t=0$ the particle is given an impulsive momentum kick $\delta p$, and we would like to calculate the subsequent evolution of the system.

The equation of motion of the particle is given by substituting for $F_{\text {ext }}$ in Eq. (21):

$$
\ddot{z}+\gamma \dot{z}+\omega_{0}^{2} z=0 \text {. }
$$

At $t=0$, immediately after the momentum kick has been applied, the position and velocity of the particle are $z(0)=0$ and $v(0)=v_{0}$, where $v_{0} \equiv \delta p / m$. We solve Eq. (53) for these initial conditions and obtain

$$
\begin{aligned}
& z(t)=\left(v_{0} / \omega_{0}\right) e^{-\gamma t / 2} \sin \omega_{0} t, \\
& v(t)=v_{0} e^{-\gamma t / 2} \cos \omega_{0} t,
\end{aligned}
$$

where for simplicity we have assumed that $\gamma \ll \omega_{0}$. We can substitute Eqs. (54) and (55) into Eqs. (8) and (9) to calculate the retarded fields $E_{r}(x, t)$ and $B_{r}(x, t)$. Because there is no incoming radiation, Eqs. (6) and (7) imply that the total fields are given by $E(x, t)=E_{r}(x, t)$ and $B(x, t)=B_{r}(x, t)$.

Let us now consider a numerical simulation of this process. The initial conditions for the scalar potential and the $B$-field are given by Eq. (46), and the initial conditions for the particle position and velocity are $z(0)=0$ and $v(0)=v_{0}$. In Fig. 2(a) we plot the particle velocity as a function of time, and in Figs. 2(b) and 2(c) we plot the $E$ and $B$ fields at time $t=420$ and $t=4 \times 10^{3}$; for comparison the fields at time $t=0$ are plotted in Fig. 1. ${ }^{15}$ For these simulations $m=2 \times 10^{3}$, $\omega_{0}=0.02$, and $v_{0}=0.3$. As expected, the particle oscillates about the origin, and the oscillations are damped due to the emission of radiation that propagates outward to the left and right.

\section{B. Braking radiation}

We now consider a free particle $\left(F_{\text {ext }}=0\right)$. As before, we will assume that there is no incoming radiation $\left(E_{\text {in }}=B_{\text {in }}\right.$ $=0$ ), so the equation of motion is

$$
\ddot{z}+\gamma \dot{z}=0 \text {. }
$$

If we use the same initial conditions as in Sec. IV A, we can solve Eq. (56) to obtain

$$
z(t)=\left(v_{0} / \gamma\right)\left(1-e^{-\gamma t}\right), \quad v(t)=v_{0} e^{-\gamma t}
$$

We can substitute Eqs. (57) into Eqs. (8) and (9) to calculate the retarded fields. As we discussed in Sec. II, in the toy model a moving particle radiates, and the emitted radiation gives rise to a drag force that damps the motion of the particle. We can view this effect as the analog to braking 

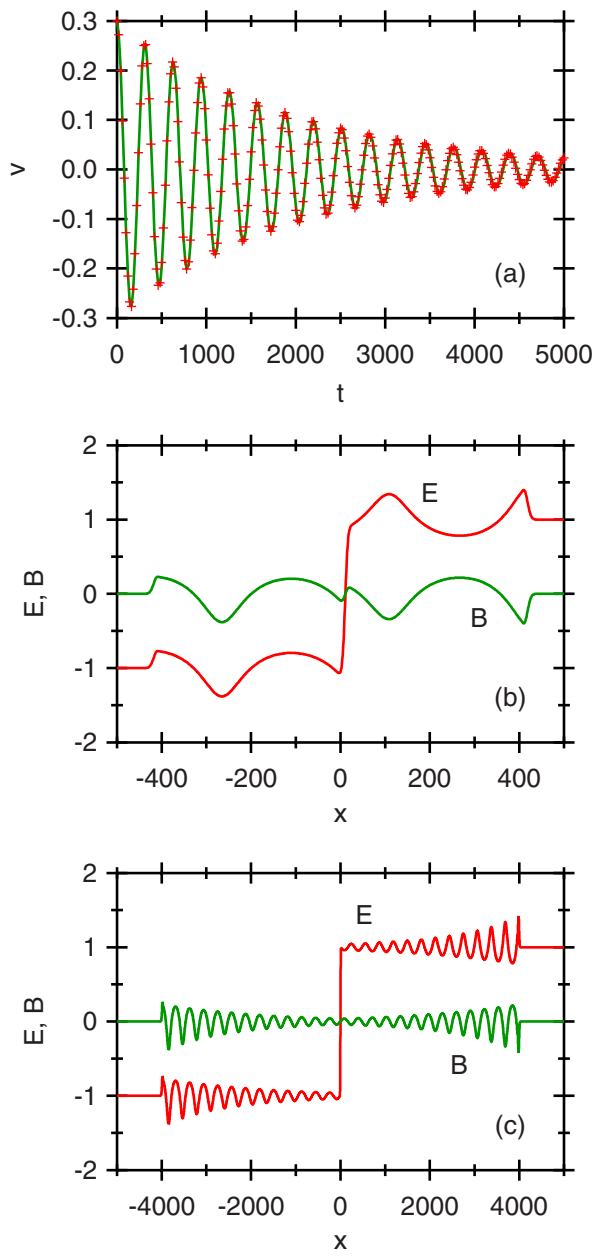

Fig. 2. Harmonically bound particle. (a) Particle velocity $v$ versus time $t$. The points are from the simulation; the solid line is the prediction given by Eq. (55). (b) $E$ and $B$ fields at time $t=420$. (c) $E$ and $B$ fields at time $t=4 \times 10^{3}$.

radiation in ordinary electrodynamics. In the latter the coupling of a charged particle to the radiation field results in a damping force that causes the particle to radiate when it accelerates. ${ }^{16}$ In the toy model the analogous damping force is proportional to the velocity of the particle, so a particle moving at a constant velocity radiates.

We now consider a numerical simulation of this process. In Fig. 3(a) we plot the particle velocity as a function of time, and in Fig. 3(b) we plot the $E$ and $B$ fields at time $t$ $=420$; for comparison, the fields at time $t=0$ are plotted in Fig. 1. ${ }^{17}$ For these simulations $m=500$ and $v_{0}=0.3$. Note that the particle velocity is exponentially damped, and the particle emits pulses of radiation that propagate outward to the left and right.

\section{Scattering}

We again consider a harmonically bound particle, but we now assume that there is an incoming wave: ${ }^{18}$

$$
E_{\text {in }}(x, t)=-\epsilon(k) B_{\text {in }}(x, t)=\operatorname{Re}\left[A e^{i(k x-\omega t)}\right] .
$$

We assume that the wave is moving to the right, so $k=\omega$. The equation of motion for the particle is
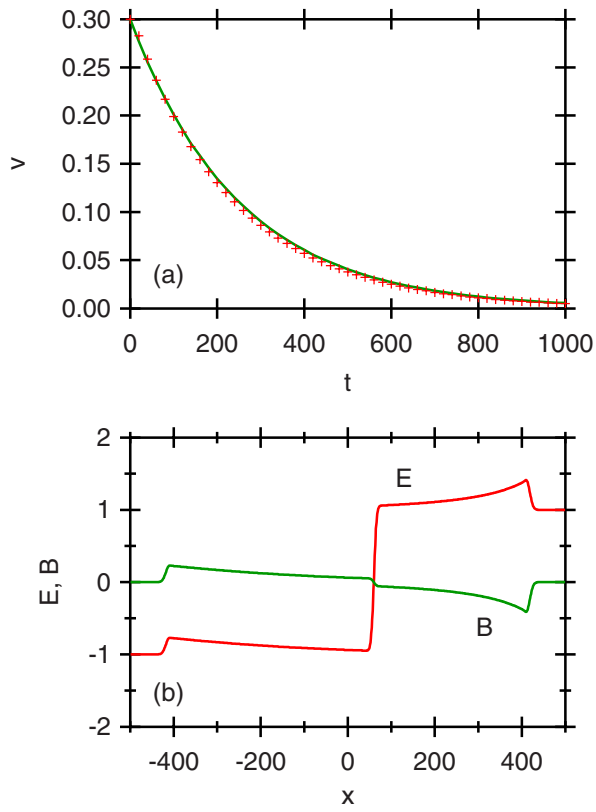

Fig. 3. Free particle. (a) Particle velocity $v$ versus time $t$. The points are from the simulation; the solid line is the prediction given by Eq. (57). (b) $E$ and $B$ fields at time $t=420$.

$$
\ddot{z}+\gamma \dot{z}+\omega_{0}^{2} z=\frac{1}{m} F_{\text {in }},
$$

where $F_{\text {in }}$ is given by Eq. (17). We assume that the displacement of the particle is much smaller than the wavelength of the incoming wave $(|k z| \ll 1)$, so we can make the dipole approximation and express $F_{\text {in }}(t)$ as

$$
F_{\text {in }}(t)=-2 g \operatorname{Re}\left[A e^{-i \omega t}\right] .
$$

If we substitute Eq. (60) into Eq. (59) and solve for $z(t)$, we find that

$$
z(t)=\operatorname{Im}\left[(A / g \omega) f(\delta) e^{-i \omega t}\right],
$$

where $\delta \equiv \omega-\omega_{0}$ is the detuning of the wave from resonance and $f(\delta) \equiv(1-i(2 \delta / \gamma))^{-1}$. (For simplicity, we have assumed that $\delta \ll \omega_{0}$.) From Eq. (61) we find that the velocity of the particle is

$$
v(t)=-\operatorname{Re}\left[(A / g) f(\delta) e^{-i \omega t}\right] .
$$

We can understand the physical meaning of $f(\delta)$ by calculating the fraction of the incident power that is transmitted across the particle. We first calculate the retarded $E$-field by substituting Eq. (62) for $v(t)$ into Eq. (8) for $E_{r}(x, t)$. In the dipole approximation $t_{r}(x, t) \simeq t-|x|$, so $E_{r}(x, t)=E_{s}(x, t)$ $+E_{\mathrm{rad}}(x, t)$, where

$$
E_{s}(x, t)=g \epsilon(x-z(t-|x|))
$$

describes the static field of the particle, and

$$
E_{\mathrm{rad}}(x, t)=g v(t-|x|)
$$

describes the emitted radiation. The total $E$-field is given by $E=E_{r}+E_{\text {in }}$, so to the right of the particle

$$
E(x, t)=E_{s}(x, t)+\operatorname{Re}\left[A(1-f(\delta)) e^{i(k x-\omega t)}\right] .
$$

Thus, the fraction of the incident power that is transmitted across the particle is ${ }^{20}$ 

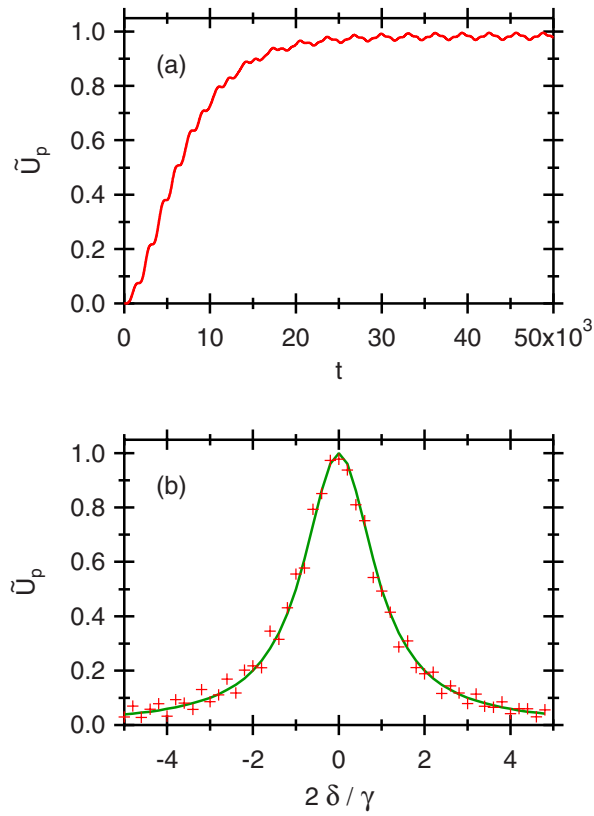

Fig. 4. Scattering from a harmonically bound particle. (a) Dimensionless energy $\widetilde{U}_{p}$ versus time $t$ for resonant driving $(\delta=0)$. (b) $\widetilde{U}_{p}$ at time $t=50$ $\times 10^{3}$ versus the dimensionless detuning parameter $2 \delta / \gamma$. The points are from the simulation; the solid line is the prediction given by Eq. (67).

$$
p(\delta)=|1-f(\delta)|^{2}=1-|f(\delta)|^{2} .
$$

From Eq. (66) we see that we can interpret $f(\delta)$ and $|f(\delta)|^{2}$ as the scattering amplitude and cross section. ${ }^{21}$

It is instructive to calculate the particle energy $U_{p}$. From Eqs. (61) and (62) we find that

$$
U_{p}=(m / 2) v^{2}+\left(m \omega_{0}^{2} / 2\right) z^{2}=(2 I / \gamma)|f(\delta)|^{2},
$$

where $I=|A|^{2} / 2$ is the intensity of the incoming radiation. Equation (67) implies that for resonant scattering $(\delta=0)$ the particle energy is $U_{p}=2 I / \gamma$. We can understand this result from a simple physical argument. The energy decay rate of the particle is $\gamma$, so the power radiated by the particle is $P=\gamma U_{p}$, and by symmetry the particle must radiate equal amounts of power to the left and to the right. ${ }^{22}$ On resonance all of the incident power is back-reflected by the particle, so the power radiated to the left must equal the incident power $I$. Thus, $P=2 I$, so $U_{p}=2 I / \gamma$.

We now consider a numerical simulation of the scattering process. We take the initial conditions of the particle to be $z(0)=v(0)=0$, and take the initial conditions for $\phi_{n}$ and $B_{n}$ to be the static fields given by Eq. (46). To simulate the incoming wave we use the boundary conditions given by Eqs. (50) and (52). We take $m=5 \times 10^{3}, \omega_{0}=0.002$, and $A=0.1$. The particle starts out at rest at the origin, so initially $U_{p}=0$. As the incoming radiation begins to drive the particle, its energy increases and it starts to radiate. Eventually $U_{p}$ reaches the steady-state value given in Eq. (67), at which time the rate at which energy is delivered by the incoming radiation balances the rate at which energy is radiated by the particle. In Fig. 4(a) we plot the dimensionless energy $\widetilde{U}_{p} \equiv(\gamma / 2 I) U_{p}$ versus time for the case of resonant driving $(\delta=0)$. As expected, the particle gradually absorbs energy from the driving field until the energy saturates at $\tilde{U}_{p}=1$. In Fig. 4(b) we vary the detuning $\delta$ of the incoming radiation, and plot $\tilde{U}_{p}$ at time $t=50 \times 10^{3}$ versus $2 \delta / \gamma$. As expected, we observe the Lorentzian response predicted by Eq. (67).

\section{Two particles}

We now consider an example with two particles. We assume that both particles have mass $m$ and charge $g$, and that they are symmetrically displaced from the origin, so if the position of particle one is $z$, the position of particle two is $-z$. Also, we assume that there is no external force or incoming radiation, so $F_{\text {ext }}=F_{\text {in }}=0$. The equation of motion for particle one is

$$
m \ddot{z}+m \gamma \dot{z}(1-\dot{z})^{-1}=-2 g E_{r 2}(z(t), t),
$$

where

$$
E_{r 2}(x, t)=\frac{g \epsilon\left(x+z\left(t_{r 2}\right)\right)}{1+v\left(t_{r 2}\right) \epsilon\left(x+z\left(t_{r 2}\right)\right)}
$$

is the retarded $E$-field generated by particle two. Here $t_{r 2}(x, t)$ is the retarded time of the event $(x, t)$ for particle two, which is defined implicitly by $t_{r 2}=t-\left|x+z\left(t_{r 2}\right)\right|$. Note that Eq. (68) is exact and does not rely on the low velocity approximation.

We will assume that for $t \leqslant 0$ the two particles are held stationary at $\pm z_{0}$ and the $E$ and $B$ fields are just the static fields of the stationary charges. At $t=0$ the particles are released and begin to move inward due to their mutual attraction. We can calculate the evolution of the system either by numerically integrating ${ }^{23}$ Eq. (68) or by simulating the coupled particle-field system. In Fig. 5(a) we plot the velocity of particle one as a function of time, and in Figs. 5(b) and 5(c) we plot the $E$ and $B$ fields at times $t=0$ and $t=8.5$ $\times 10^{3}$. For these simulations $m=5 \times 10^{3}$ and $z_{0}=500$. We see that the mutual attraction of the particles causes them to oscillate, and the oscillations are damped due to the emission of radiation.

\section{ADVANCED TOPICS}

So far we have assumed that the extended particle is small enough that it is well approximated by the point particle equation of motion Eq. (20). As the particle size increases this approximation breaks down and a new equation of motion is needed. We can derive an exact equation of motion for an extended particle by substituting the $E$-field decomposition given by Eq. (6) into the force law for an extended particle given by Eq. (29). We find that $F_{f}=F_{\text {in }}+F_{r}$, where

$$
F_{\text {in }}(t)=-2 g \int \rho(x, t) E_{\text {in }}(x, t) d x
$$

is the force exerted on the particle by the incoming radiation, and

$$
F_{r}(t)=-2 g \int \rho(x, t) E_{r}(x, t) d x
$$

is the radiation reaction force exerted on the particle by its own retarded field. One can show that the retarded $E$-field generated by an arbitrary charge distribution $\rho(x, t)$ is given by 

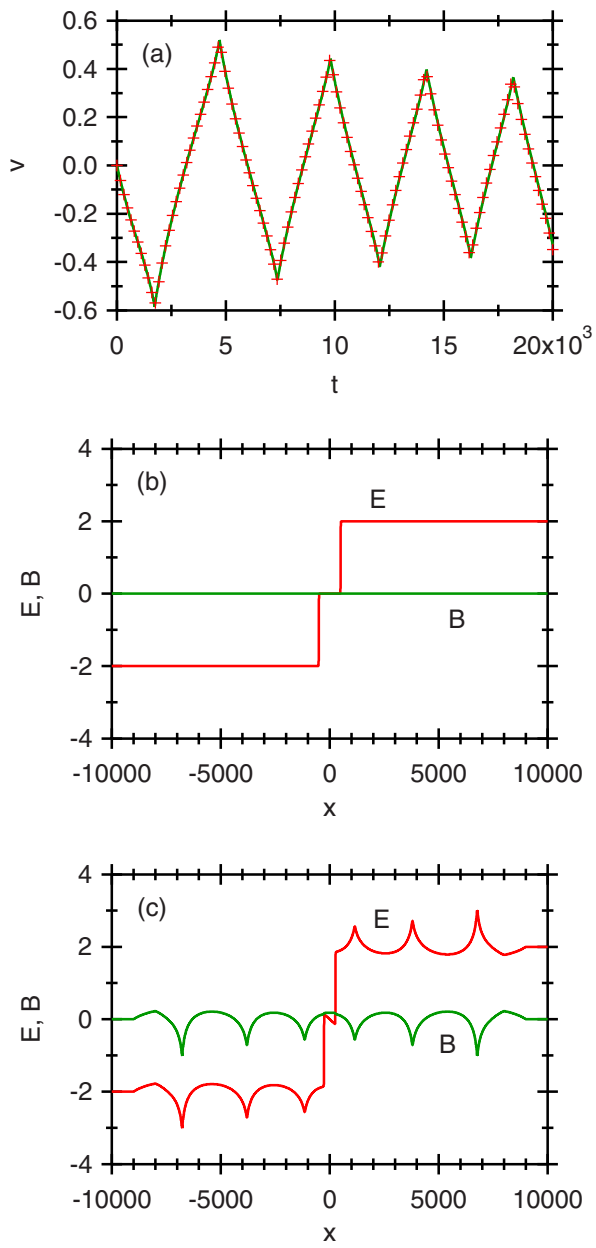

Fig. 5. (a) Velocity $v$ of particle one versus time $t$. The points are from the simulation; the solid line is obtained by numerically integrating Eq. (68). (b) $E$ and $B$ fields at time $t=0$. (c) $E$ and $B$ fields at time $t=8.5 \times 10^{3}$.

$$
E_{r}(x, t)=\int \epsilon(x-y) \rho(y, t-|x-y|) d y .
$$

If we substitute Eq. (72) into Eq. (71), substitute for $\rho(x, t)$ using Eq. (27), and perform the integral over $x$, we find that

$$
F_{r}(t)=-\frac{m \gamma}{2 \sqrt{\pi} \sigma} \int e^{-(z(t)-z(t-|s|)-s)^{2} / 4 \sigma^{2}} \epsilon(s) d s .
$$

Thus, the exact equation of motion for a spatially extended particle is

$$
m \ddot{z}=F_{\text {ext }}+F_{\text {in }}+F_{r},
$$

where $F_{\text {ext }}$ is the externally imposed force, $F_{\text {in }}$ is given by Eq. (70), and $F_{r}$ is given by Eq. (73).

It is interesting to compare the radiation reaction force given in Eq. (73) with the radiation reaction force for a point particle given in Eq. (19). For a point particle $F_{r}(t)$ depends only on the state of the particle at time $t$, but for a spatially extended particle $F_{r}(t)$ depends on the state of the particle at times $t^{\prime} \leqslant t$.

We now consider when an extended particle can be approximated as a point particle. For a sufficiently small particle we can obtain a good approximation to the radiation reaction force given in Eq. (73) by expanding $z(t-|s|)$ in $|s|$

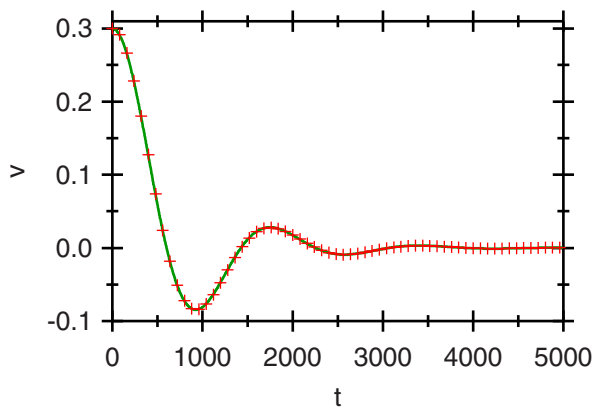

Fig. 6. Particle velocity $v$ versus time $t$. The points are from the simulation of a spatially extended particle; the solid line is obtained by numerically integrating Eq. (74).

and retaining only the first-order term: $z(t-|s|) \simeq z(t)$ $-v(t)|s|$. If we make this approximation and perform the integral, we find that Eq. (73) reduces to the radiation reaction force for a point particle given in Eq. (19). The first-order approximation to $z(t-|s|)$ is justified if $\sigma^{n} v^{(n)} \ll 1$ for $n>0$, where $v^{(n)}(t) \equiv d^{n} v(t) / d t^{n}$. When these conditions are met, the extended particle can be approximated as a point particle.

To show how the behavior of an extended particle differs from that of a point particle, we repeat the numerical simulation of a free particle that was described in Sec. IV B, but we now take the particle size to be $\sigma=250$. In Fig. 6 we plot the particle velocity as a function of time; Fig. 6 should be compared to Fig. 3(a), which shows the analogous graph for a particle of size $\sigma=5$. For Fig. 3(a) the velocity is exponentially damped, but for Fig. 6 the velocity oscillates as it damps. The oscillations are due to the fact that one segment of the extended particle can act on the field, which then acts back on a different segment of the particle at a later time.

\section{ACKNOWLEDGMENT}

The author would like to thank Andreea Boca for preparing the figures.

\section{APPENDIX A: ELECTRODYNAMICS IN $(1+1)$ DIMENSIONS}

We give here a brief overview of electrodynamics in $(1+1)$ dimensions, which describes a charged particle coupled to a single field $E(x, t) .{ }^{24}$ The field equations for $E(x, t)$ are

$$
\partial_{x} E(x, t)=2 \rho(x, t), \quad \partial_{t} E(x, t)=-2 J(x, t) .
$$

The charge density $\rho(x, t)$ and current density $J(x, t)$ are given by

$$
\rho(x, t)=q \delta(x-z(t)), \quad J(x, t)=v(t) \rho(x, t),
$$

where $z(t)$ and $v(t)$ are the position and velocity of the particle at time $t$ and $q$ is its charge. Note that $\rho(x, t)$ and $J(x, t)$ satisfy the conservation law

$$
\partial_{t} \rho(x, t)+\partial_{x} J(x, t)=0 .
$$

The equation of motion for the particle is

$$
m \ddot{z}=F_{\text {ext }}+F_{f},
$$

where $F_{\text {ext }}$ is an externally imposed force and 


$$
F_{f}(t)=2 q E(z(t), t)
$$

is the force that the $E$-field exerts on the particle. Equations (A1)-(A5) give a complete description of electrodynamics in $(1+1)$ dimensions. There are a number of ways of motivating these equations: for example, they can be obtained by writing Maxwell's equations in tensor form and then generalizing these tensor equations to $(1+1)$ dimensions. ${ }^{25}$ Equations (A1) and (A5) can also be obtained from Maxwell's equations and the Lorentz force law by imposing translational symmetry in the $\hat{y}$ and $\hat{z}$ directions.

In some respects electrodynamics in $(1+1)$ dimensions is a closer analog to ordinary electrodynamics than the toy model we have considered. For example, one can show that electrodynamics in $(1+1)$ dimensions is Lorentz invariant and gives a repulsive force between charges of the same sign. However, it differs from ordinary electrodynamics in some important respects. For example, the $E$-field can be viewed as the analog of the electric field, but there is no analog to the magnetic field. Also, there is no radiation. To see why, note that we can explicitly solve the field Eqs. (A1) for the $E$-field:

$$
E(x, t)=E_{0}+q \epsilon(x-z(t)),
$$

where $E_{0}$ is a background $E$-field that is constant in time and uniform in space. Equation (A6) shows that the $E$-field is uniquely determined by the particle position and the value of $E_{0}$, and thus does not support radiation. Because the goal of this paper is to illustrate radiative phenomena, we have chosen to use the toy model defined by Eqs. (1)-(5) instead of electrodynamics in $(1+1)$ dimensions.

\footnotetext{
a)Electronic mail: boozer@caltech.edu

${ }^{1}$ The toy model that we will consider is not the same as electrodynamics in $(1+1)$ dimensions, which is discussed in Appendix A.

${ }^{2}$ The computer program used to perform the simulations will be provided upon request.

${ }^{3}$ A complete description of the toy model is given in A. D. Boozer, "A toy model of electrodynamics in $(1+1)$ dimensions," Eur. J. Phys. 28, 447464 (2007). Derivations of the various results we cite regarding the toy model can be found there.

${ }^{4}$ We have chosen a system of units such that the speed at which waves are propagated by the field is equal to one.

${ }^{5}$ Note that there is a minus sign on the right-hand side of Eq. (5), but there is no analogous minus sign for the Lorentz force law. Also, the Lorentz force law contains a term that involves the magnetic field, but there is no corresponding term in Eq. (5). The reason for choosing the force law given in Eq. (5) is explained in Ref. 3.

${ }^{6}$ This is not the only way of performing the decomposition, but it is the most useful for our present purposes. See A. D. Boozer, "Retarded potentials and the radiative arrow of time," Eur. J. Phys. 28, 1131-1143
}

(2007).

${ }^{7}$ Here $\epsilon(x)$ is the sign function, defined such that $\epsilon(x)=1$ if $x>0, \epsilon(x)$ $=0$ if $x=0$, and $\epsilon(x)=-1$ if $x<0$.

${ }^{8}$ The retarded fields of a charged point particle in ordinary electrodynamics are derived in D. J. Griffiths, Introduction to Electrodynamics, 2nd ed. (Prentice Hall, Englewood Cliffs, NJ, 1989), Sec. 9.2.2.

${ }^{9}$ Expressions for the energy and momentum density of the field are derived in Ref. 3. By using these expressions one can show that total energy and momentum of the coupled particle-field system are conserved, and that the energy lost by the particle to radiation damping is equal to the energy gained by the field.

${ }^{10} \mathrm{~A}$ detailed comparison of the toy model and ordinary electrodynamics is given in Ref. 3.

${ }^{11}$ For the toy model there is a preferred reference frame in which the equations of motion are valid, and a particle moving with respect to this preferred frame feels a drag force that is given by Eq. (19).

${ }^{12}$ To simulate incoming radiation we replace Eq. (51) by Eq. (52) as described in Sec. III B.

${ }^{13}$ The analogous problem in ordinary electrodynamics is discussed in Ref. 8, pp. 434-435, and in Ref. 14.

${ }^{14}$ J. D. Jackson, Classical Electrodynamics, 2nd ed. (John Wiley \& Sons, New York, 1975), Sec. 17.7.

${ }^{15}$ Pictures of the fields for a charge undergoing simple harmonic motion in ordinary electrodynamics are given in R. Y. Tsien, "Pictures of dynamic electric fields," Am. J. Phys. 40(1), 46-56 (1972) and R. H. Good, "Dipole radiation: A film,” Am. J. Phys. 49(2), 185-187 (1981). An animation of the fields for dipole radiation in ordinary electrodynamics is discussed in J. W. Belcher and S. Olbert, "Field line motion in classical electromagnetism," Am. J. Phys. 71(3), 220-228 (2003).

${ }^{16}$ Braking radiation in ordinary electrodynamics is discussed in Ref. 8, pp. 430-431, and in Ref. 14, Sec. 15.

${ }^{17}$ Pictures of the fields for braking radiation in ordinary electrodynamics are given in J. C. Hamilton and J. L. Schwartz, "Electric fields of moving charges: A series of four film loops," Am. J. Phys. 39(12), 1540-1542 (1971) and R. H. Good, "Dipole radiation: Simulation using a microcomputer," Am. J. Phys. 52(12), 1150-1151 (1984).

${ }^{18}$ The analogous problem for ordinary electrodynamics is discussed in Ref. 14, Sec. 17.8

${ }^{19}$ From Eqs. (61) and (62) we see that $|k z| \ll 1$ implies $|v| \ll 1$, so the dipole approximation implies the low-velocity approximation.

${ }^{20}$ We have used the fact that $\operatorname{Re} f=|f|^{2}$. This relation is the toy-model ana$\log$ to the optical theorem of ordinary electrodynamics (see Ref. 14, Sec. 9.14).

${ }^{21}$ Note that in $(1+1)$ dimensions the scattering cross section is a dimensionless quantity ranging from 0 to 1 that gives the fraction of the incident power that is back-reflected from the scattering site.

${ }^{22}$ Note that although the particle radiates power to the right, on the righthand side of the particle the radiated field exactly cancels the incident field $\left(E_{\text {rad }}(x, t)=-E_{\text {in }}(x, t)\right)$, so there is no radiation in the total field $E(x, t)$.

${ }^{23}$ Because $E_{r 2}(z(t), t)$ depends on $z\left(t_{r 2}\right)$, we must keep a record of the past trajectory of the particle to perform the numerical integration.

${ }^{24}$ Electrodynamics in $(1+1)$ dimensions is discussed in many places. See for example, H. Galić, "Fun and frustration with hydrogen in a $1+1$ dimension,” Am. J. Phys. 56(4), 312-317 (1987).

${ }^{25}$ This approach is discussed in Ref. 24, Sec. II. 